\title{
A Simulation Tool for the Performance Evaluation of Large-scale RF-Mesh Networks for Smart Grid and IoT Applications
}

\author{
Filippo Malandra ${ }^{1}$ and Brunilde Sansò ${ }^{1}$ \\ Ecole Polytechnique de Montreal, Montreal, Québec, Canada \\ Department of Electrical Engineering \\ filippo.malandra@polymtl.ca
}

\begin{abstract}
Driven by the need of robust, cost-effective, and ready-to-use solutions to connect wirelessly thousands to million of nodes, an increasing number of applications such as Smart Grids and IoT networks use large-scale Wireless Mesh Networks as transmission support. Tools and methodologies to study the performance of such systems are constantly sought and become fundamental in the feasibility assessment of the high number of possible applications. In this paper, a simulation tool is proposed to study the performance of a particular kind of Wireless Mesh Network, based on the RF-Mesh technology. The modular nature of the implemented tool allows for a smooth extension to the performance analysis of other types of Wireless Mesh Networks using technologies similar to RF-Mesh. The tool was implemented in the context of a large-scale Smart Grid AMI (Advanced Metering Infrastructure) system. The tool, coded in Java and Python, considers different types of traffic and provides the end-to-end delay and several other performance indexes of large scale (i.e., with several thousand nodes) instances in the order of minutes.
\end{abstract}

\section{Introduction}

Information Communication Technology (ICT) systems play a key role in the development of smart cities, smart grids, and the Internet of Things (IoT). An increasing number of applications for those systems need a solid and steady communication infrastructure. Those applications require the communication of a large number of nodes (e.g., smart meter, smart appliances, sensors) over extended and heterogeneous areas. The need for easy and quick deployment, the harsh and variegated propagating environments, and the large number of nodes makes popular the choice of Wireless Mesh Networks for IoT and smart grid infrastructures. Among the adopted technologies, the RF-Mesh seems to be one of the most popular in smart grids Advanced Metering Infrastructures (AMIs) thanks to its ease of implementation, the limited cost of the equipment, and the use of the free and unlicensed ISM bandwidth centered on $900 \mathrm{MHz}$.

When it comes to the definition of the applications to be enabled by RF-Mesh networks the following questions arise: up to how many nodes can be connected? What is the bandwidth utilization? What is the average delay at node level? 
What are the limits and the advantages brought by this technology? Given the large-scale nature of these systems and the fact that there are not many public details available (they are often sold as black boxes to utility companies), a thorough end-to-end performance evaluation that answers the questions above is often difficult to achieve.

The current literature on the performance evaluation of RF-Mesh systems reflects three main approaches: 1) analytic studies, 2) field trials and 3) stochastic simulations. The first approach, used for example in $[1,2]$, consists in analytically computing the packet collision probability by exploiting fundamental properties of wireless communications. The approach proved computational efficient and flexible but requires some assumptions that reduce its accuracy. For instance, the modelling of the Frequency Hopping Spread Spectrum (FHSS) protocol is very simple and the computation of the delay does not take into account the waiting time of packets in the buffer or the retransmission probability at each time slot. Field trials are implemented in deployed systems where packet delays and other sensitive parameters are measured. This approach permits analyzing details that are difficult to be included in the analytic formulations but the implementation costs are high and the settings are often limited so that it is difficult to study other kinds of instances and scenarios. Stochastic simulations lie in between the two aforementioned approaches. They are based on the development of software that tries to faithfully reproduce the operation of deployed RF-Mesh systems. Software programming allows to go beyond the limits of analytic modelling, enabling the representation of complex implementation details. Moreover, the same simulator can be applied to different instances and scenarios providing a larger flexibility with respect to field trials. For this reason, in the present work, we decided to use stochastic simulations in order to evaluate the performance of large-scale RF-Mesh systems for smart grids.

Several performance frameworks and platforms are currently used in communications simulation. The most widespread tools are NS-2 and NS-3 (e.g., $[3,4,5])$, Castalia (e.g., [6]), OPNET (e.g., [7, 8, 9, 10, 11]), OMNET++ (e.g., $[12],[13])$ and the Contiki OS and its network emulator framework COOJA (e.g., $[14,15])$. Despite the large number of off-the-shelf simulation packages, we could not find any module specifically dedicated to the RF-Mesh technology and capable to evaluate the performance of large scale systems in a reasonable time. Therefore, we decided to build from a scratch a new simulator with increased computational efficiency by customizing its design around the peculiarities of the RF-Mesh technology.

In this paper, we propose a thorough and flexible simulation tool whose main objective is to shed some light on the performance of RF-Mesh systems, highlighting both the advantages and the limits of this particular technology. The tool was tested in the context of a large AMI system for smart grids. It proved to be very useful in the definition of the type of applications that can be enabled, based on its communication requirements.

The reminder of this document is structured as follows. Section 2 describes the system under consideration. Section 3 provides some implementation de- 
tails about the simulator. Section 4 shows some numerical results obtained with the simulation tool. Section 5 includes concluding remarks and possible future extensions of the present contribution.

\section{Description of the RF-Mesh system}

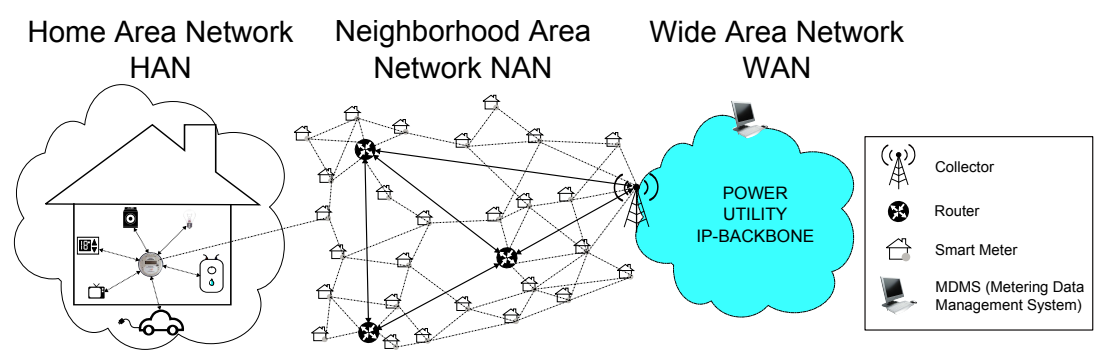

Fig. 1. Architecture of the RF-Mesh AMI.

Fig. 1 shows that RF-Mesh AMIs are characterized by a layered architecture composed of: (i) Home Area Network (HAN), (ii) Neighborhood Area Network (NAN), (iii) Wide Area Network (WAN). The performance analysis presented in this paper is centered on the NAN layer, that is a mesh network whose main objective is to connect the smart meters to one or more data collectors. The topology is completed by wireless routers, which are used to extend wireless coverage. Smart meters produce packets directed to the data collectors, whereas routers are only used as intermediate nodes.

The main features of a RF-Mesh AMI to be taken into account in the simulation framework can be summarized as follows.

Large number of nodes Smart meters are the most numerous devices in the topology: their number ranges from roughly a thousand per neighborhood in a rural environment up to tens of thousands in densely populated urban environments. The high number of nodes affects the size of the simulations, increasing the computational burden as the network size increases.

Low throughput Several factors (e.g., interference, use of public frequency bandwidth, low quality devices) make the nominal rates of the wireless link low (e.g., $19.2 \mathrm{kbps}$ for links between routers and data collectors, and $9.6 \mathrm{kbps}$ for other links).

Black-box nature of the system and undisclosed features AMI systems are often sold to power utilities as real black boxes for which very technical details are only provided under strict non-disclosure agreements. Moreover, once installed, smart meter data is considered very sensitive. As a result, some of the 
characteristics of RF-Mesh AMIs are not publicly disseminated, despite their importance in the definition of network performance. In the process of designing our simulation framework, we did some reverse engineering and carefully scanned any publicly available piece of information to validate the simulator, while making the modules as flexible as possible to encompass many different types of undisclosed features.

MAC layer Two packets that are simultaneously transmitted on the same wireless channel create a collision. Wireless systems react in different ways after a collision, depending on the implemented Media Access Control (MAC) layer protocol. For the given simulation framework we considered a smart-meter communication system using a MAC layer based on time-slotted ALOHA according to which time is divided into time slots and a node is allowed to transmit at the beginning of each time slot. When a node has a packet ready, it waits until the beginning of the following time slot to transmit it. If the correct reception of a given packet $i$ by node $A$ is prevented by a concurrent transmission on the same time slot, node $A$ is backlogged and will attempt a retransmission of packet $i$ in one of the following time slots with probability $p_{r}$. Packets retransmission degrades the overall performance because it increases system delay.

Wireless interference issues AMI RF transmissions take place in a free unlicensed band, leading to severe interference issues. Thus, even though interference can come from external devices, such as cordless phones, we neglected those sources and only modeled the interference from the same RF mesh. The presence of thousands of potential simultaneous transmitters in a given area entailed the adoption of strong measures to mitigate the effect of interference. Among the spread spectrum techniques off-the-shelf, FHSS was chosen for its capacity to reduce co-channel interference. The mechanism of FHSS is based on the subdivision of the bandwidth in a number $\theta$ of channels. The same sequence of channels is used by each node, opportunely shifted over time. This technique greatly reduces the impact of interference on performance and facilitates the communications even in very densely populated environments.

Network layer An active role in the dynamics of a mesh system is played by the choice of the routing protocol, that can greatly impact the network performance. In this simulator, the so called Layer-based routing is used, based on hierarchical division of the nodes: every node is assigned to a layer (i.e., collector is in layer 0 , its neighbors in layer 1 and so on). The downlink path is decided by the collector, based on information collected in the layer formation phase. On the other hand, each smart meter transmits packets in uplink direction using one of its neighbors in the upper layer. The advantage of this mechanism is that it is very simple on the smart meter side. 


\section{Simulation framework}

The operation of the simulator at hand can be subdivided in three different phases: Initialization (see Section 3.1), Simulation(see Section 3.2), and Results analysis(see Section 4).

\subsection{Initialization phase}

The objective of the initialization phase is to perform all the preliminary operations to the subsequent Simulation phase. First, one of the previously generated topology is chosen or a new one is created: topology is defined by a list of nodes (identified by an ID and GPS coordinates) and links between them (chosen according to fixed covering rays that depend on the propagating environment). Then, the user is given the possibility to tune some relevant parameters (i.e., simulation horizon, packet generation rate, buffer size) according to his/her needs. Finally, the routing mechanism is defined, based on bidirectional shortest paths from each smart meter to the data collector.

\subsection{Simulation phase}

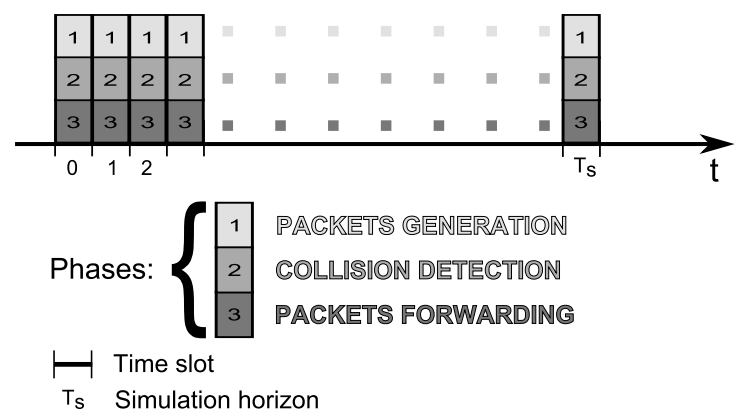

Fig. 2. Scheme of the simulation phase.

The Simulation phase, schematized in Figure 2, represents the core of the implemented tool. The simulation horizon is subdivided in time steps of fixed duration $\tau$ and three main operations are performed during each time step:

- packet generation: smart meters and data collectors generate packets according to a Poisson distribution whose parameters are defined in the Initialization phase.

- collision detection: for each of the transmitted packets a check is performed for possible collisions with other simultaneous transmissions in the same transmission range of the receiving node. Involved packets are flagged for collision.

- packet forwarding: flagged packets are to be re-transmitted in one of the following time slots, whereas all the others are forwarded to their destination. 


\section{Numerical results}

Numerical results include delay and activity time evaluation in a scenario obtained using publicly available data.

Mansonville, was one of the three sites used for a pilot project of smart meter installation in Québec [16]. Given that some of the data was available, we used it to build our set of experiences. The topology includes 1 data collector, 115 routers, and 3300 smart meters. The location of routers and collectors were derived from [16]; the location of smart meters, not included in the previous document, were derived by using an API provided by Bing Maps.

Once the topology was created, three traffic streams were defined, $\alpha, \beta$ and $\gamma$, in order to represent the communications required by some smart grid applications:

$\alpha$ Poisson distributed packet generation from each smart meter to the data collector (with mean parameter $\lambda_{u}$ )

$\beta$ Poisson distributed packet generation from the data collector to each smart meter (with mean parameter $\lambda_{d}$ )

$\gamma$ broadcast transmission from the collector to all the smart meter at a predetermined time of the day.

40 traffic scenarios were defined according to different values of $1 / \lambda_{u}(0.125$, $0.25,0.5$, and 1 hour), and of $1 / \lambda_{d}(0.5,1,2,3$, and 4 hours). In half of the scenarios only $\alpha$ and $\beta$ were considered, whereas in the other half a daily broadcast transmission (i.e., $\gamma$ ) was also scheduled. FOr all simulations, the time horizon was $T_{s}=604800 \mathrm{~s}$ (i.e., 7 days) and the time-slot duration was $\tau=0.7 \mathrm{~s}$. In order to verify the consistency of the results, a confidence interval analysis is proposed, based on 50 independent runs for every traffic scenarios. For all the performance indexes, we report the average values and the $95 \%$-confidence intervals.

\subsection{Delay profile}

The delay was calculated as the difference of the arrival time at the destination and the generation time at the source for each packet. In Table 1, the 95\%confidence intervals of delay according to different levels of $1 / \lambda_{u}$ and $1 / \lambda_{d}$ are reported in the form $\mu \pm \sigma$ : the low values of $\sigma$ obtained with respect to $\mu$ prove the consistency of the simulator. It is observed that uplink and downlink delays increase as the mean packet generation time decreases and respectively range from $33.92 \mathrm{~s}$ to $38.39 \mathrm{~s}$ and from $37.46 \mathrm{~s}$ to $43.98 \mathrm{~s}$. Moreover, the delay results to be higher in downlink than in the uplink direction.

The average delay in uplink and downlink in the traffic scenario with $1 / \lambda_{d}=0.5 \mathrm{~h}$ and $1 / \lambda_{u}=0.125 \mathrm{~h}$ is reported in Figure 3, through heat-maps. On these maps, the collector is represented by a blue circle and the routers by red stars. Each smart meter is represented by a spot whose color depends on its average delay, according to the color-bar reported on the right of each figure. We can notice that the average downlink delay is larger than the average 

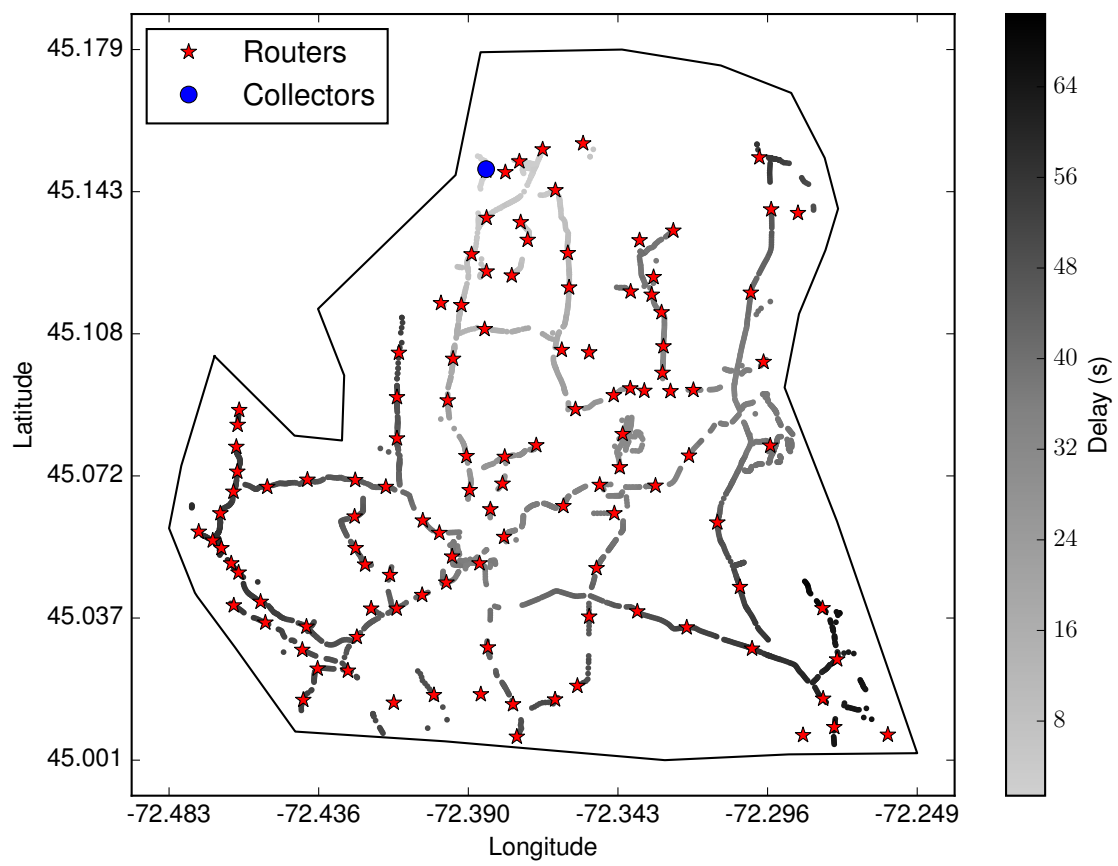

(a) uplink

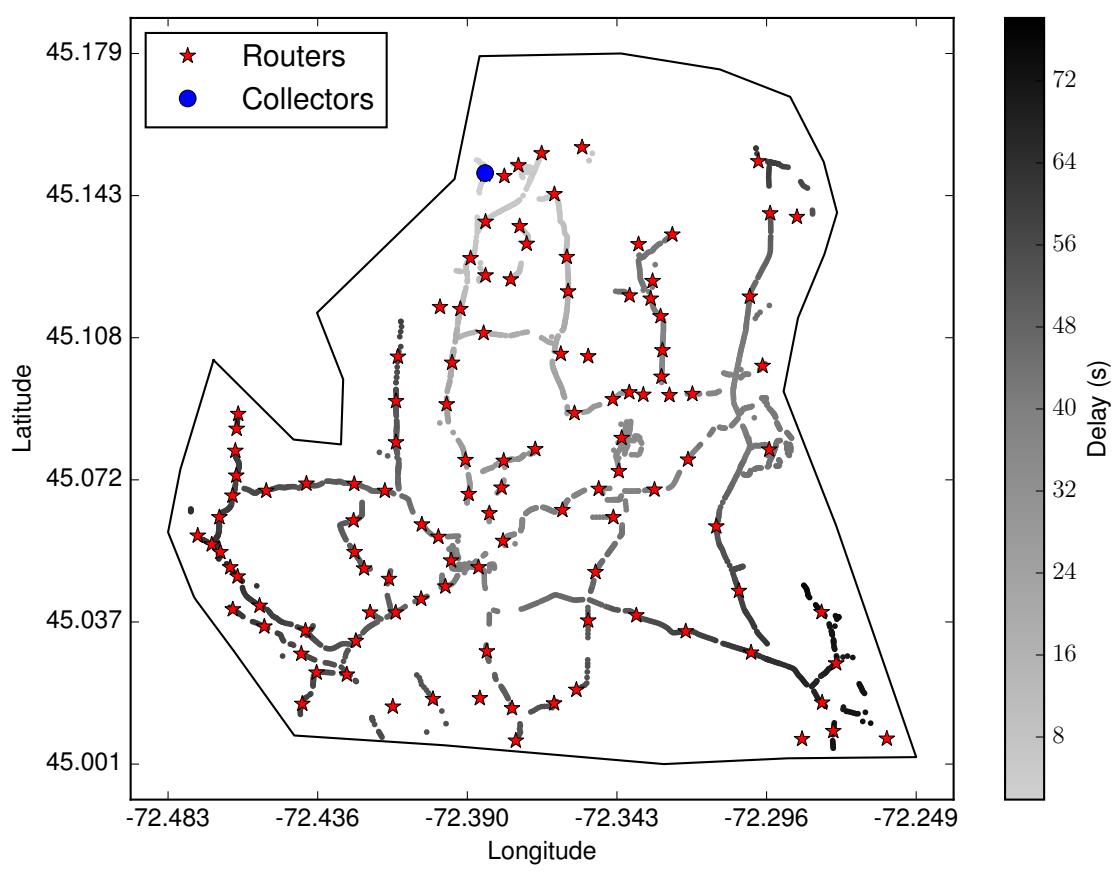

(b) downlink

Fig. 3. Heat-map of the delay in the scenario with $1 / \lambda_{d}=0.5 \mathrm{~h}$ and $1 / \lambda_{u}=0.125 \mathrm{~h}$. 
Table 1. Average delay according to different levels of $1 / \lambda_{u}$ (rows) and $1 / \lambda_{d}$ (columns).

\begin{tabular}{c|cccc}
\hline & \multicolumn{4}{|c}{ Uplink delay ( $\alpha$ traffic) (sec) } \\
$\begin{array}{c}1 / \lambda_{d} \\
\text { (hours) }\end{array}$ & \multicolumn{4}{|c}{$1 / \lambda_{u}$ (hours) } \\
\hline 4 & $\mathbf{3 3 . 9 2} \pm 0.35$ & $33.93 \pm 0.29$ & $34.19 \pm 0.39$ & $34.94 \pm 0.54$ \\
3 & $34.29 \pm 0.36$ & $34.41 \pm 0.30$ & $34.67 \pm 0.34$ & $35.50 \pm 0.54$ \\
2 & $35.01 \pm 0.35$ & $35.11 \pm 0.35$ & $35.42 \pm 0.43$ & $36.18 \pm 0.52$ \\
1 & $36.15 \pm 0.34$ & $36.24 \pm 0.36$ & $36.60 \pm 0.40$ & $37.44 \pm 0.55$ \\
0.5 & $37.07 \pm 0.41$ & $37.18 \pm 0.34$ & $37.48 \pm 0.36$ & $\mathbf{3 8 . 3 9} \pm 0.49$ \\
\hline & \multicolumn{4}{|c}{ Downlink delay $(\beta$ traffic) $(\mathrm{sec})$} \\
$1 / \lambda_{d}$ & \multicolumn{4}{|c}{$1 / \lambda_{u}$ (hours) } \\
$($ hours $)$ & 1 & 0.5 & 0.25 \\
\hline 4 & $\mathbf{3 7 . 4 6} \pm 0.38$ & $38.89 \pm 0.42$ & $40.30 \pm 0.46$ & $41.53 \pm 0.50$ \\
3 & $37.73 \pm 0.42$ & $39.11 \pm 0.41$ & $40.55 \pm 0.51$ & $41.72 \pm 0.56$ \\
2 & $38.05 \pm 0.41$ & $39.45 \pm 0.43$ & $40.84 \pm 0.49$ & $42.16 \pm 0.58$ \\
1 & $38.86 \pm 0.51$ & $40.16 \pm 0.44$ & $41.65 \pm 0.48$ & $43.01 \pm 0.54$ \\
0.5 & $39.72 \pm 0.37$ & $41.07 \pm 0.47$ & $42.60 \pm 0.51$ & $\mathbf{4 3 . 9 8} \pm 0.52$ \\
\hline
\end{tabular}

uplink delay. This is due to the presence of broadcast transmission, which is in the downlink direction. Despite slightly different average values, the uplink and downlink delays smoothly increase at a similar pace as the distance from the collector increases. The aforementioned figure does not highlight the presence of congestioned area in the topology under consideration.

\subsection{Activity time}

Another relevant parameter in the performance analysis is the activity time $\chi(i)$ of smart meters, representing the percentage of time slots in which smart meter $i$ is transmitting. This parameter is useful to characterize the amount of wireless transmissions generated by the smart meter communication system under consideration. The analysis was driven by the public perceived impact of wireless transmissions on human health. 95\%-confidence intervals for $\chi$ are reported in Table 2. It is important to remark that the average activity times for smart meters are in every scenario between 0.3 and $0.9 \%$, very small values if compared to other wireless devices (e.g., WiFi routers, smart-phones, smart TVs).

\subsection{Computational time}

RF-Mesh systems are characterized by a thousands of nodes, therefore the computational time might seriously undermine the performance analysis and needs to be seriously considered. In our simulations, however, the average computational time to study 3416-node instances over a simulated time of 7 days was of 344 seconds, on a machine with a processor $\operatorname{Intel}(R) X e o n(R) X 5675$ working 
Table 2. Average activity times for the smart meters.

\begin{tabular}{c|cccc}
\hline $\begin{array}{c}1 / \lambda_{d} \\
\text { (hours) }\end{array}$ & 1 & \multicolumn{4}{|c}{$1 / \lambda_{u}$ (hours) } \\
\hline 4.0 & $\mathbf{3 . 2 2 e - 3} \pm 2.32 \mathrm{e}-5$ & $4.54 \mathrm{e}-3 \pm 4.26 \mathrm{e}-5$ & $6.26 \mathrm{e}-3 \pm 4.96 \mathrm{e}-5$ & $7.55 \mathrm{e}-3 \pm 1.05 \mathrm{e}-4$ \\
3.0 & $3.39 \mathrm{e}-3 \pm 2.82 \mathrm{e}-5$ & $4.69 \mathrm{e}-3 \pm 3.75 \mathrm{e}-5$ & $6.41 \mathrm{e}-3 \pm 6.03 \mathrm{e}-5$ & $7.71 \mathrm{e}-3 \pm 1.08 \mathrm{e}-4$ \\
2.0 & $3.65 \mathrm{e}-3 \pm 3.25 \mathrm{e}-5$ & $4.94 \mathrm{e}-3 \pm 3.87 \mathrm{e}-5$ & $6.64 \mathrm{e}-3 \pm 4.34 \mathrm{e}-5$ & $7.92 \mathrm{e}-3 \pm 9.53 \mathrm{e}-5$ \\
1.0 & $4.14 \mathrm{e}-3 \pm 4.72 \mathrm{e}-5$ & $5.40 \mathrm{e}-3 \pm 5.26 \mathrm{e}-5$ & $7.07 \mathrm{e}-3 \pm 5.99 \mathrm{e}-5$ & $8.34 \mathrm{e}-3 \pm 1.14 \mathrm{e}-4$ \\
0.5 & $4.57 \mathrm{e}-3 \pm 4.18 \mathrm{e}-5$ & $5.80 \mathrm{e}-3 \pm 5.36 \mathrm{e}-5$ & $7.45 \mathrm{e}-3 \pm 6.82 \mathrm{e}-5$ & $\mathbf{8 . 6 9 e}-3 \pm 1.10 \mathrm{e}-4$ \\
\hline
\end{tabular}

at $3.07 \mathrm{GHz}$. This is a remarkable result because it shows that it is possible to analyze large-scale systems in a considerably short time. It is also worth highlighting that the elapsed time of 344 seconds amounts to $0.057 \%$ of the total simulated time of $604800 \mathrm{~s}$ : on average, $0.57 \mathrm{~ms}$ are necessary to simulate $1 \mathrm{~s}$ of operation of the network.

\section{Conclusion}

The applications for Smart cities, Smart Grids and IoT are proliferating and their growth is expected to significaly increase in the future. Therefore, it is essential for the actors involved (e.g., power utilities) to use tools able to study the performance of large scale communication systems, in order to assess the feasibility of smart grid applications. In this paper, a simulation tool to study the performance of large-scale Wireless Mesh Networks, based on the RF technology was presented and analyzed within the context of a smart-grid AMI system,

The tool proved to be computationally efficient, allowing to analyze instances with several thousand nodes in just few minutes. Moreover, the tool provides flexibility in the choice of the parameters defining the traffic and the topology, entailing customized solutions to the user. The simulator can handle pre-built topologies chosen by the user or it can be used in combination with an optimization model to select the position of routers and data collectors.

Numerical results showed the wide range of analyses that can be carried out with the tool at hand. This allows to gain insight into the system under consideration. In the presented scenario, a degradation of the performance was observed when using broadcast traffic, and, in particular, the delay in the downlink appears to be larger, on average, than in the uplink. The use of the activity time parameter enriches the analysis for massive smart meters installation.

\section{References}

1. F. Malandra and B. Sansò, "Analytical performance analysis of a large-scale RFmesh smart meter communication system," in Proc. IEEE Innovative Smart Grid Technologies Conference (ISGT 2015), Feb 2015. 
2. F. Malandra and B. Sansò, "PeRF-Mesh: a performance analysis tool for large scale RF-Mesh-based smart meter networks with FHSS," in Proc. IEEE Int. Conf. on Smart Grid Communications (SmartGridComm), Miami, USA, Nov. 2015.

3. A. AlMajali, A. Viswanathan, and C. Neuman, "Analyzing resiliency of the smart grid communication architectures under cyber attack," in Proc. 5th Workshop on Cyber Security Experimentation and Test, 2012.

4. M. Yigit, E. A. Yoney, and V. C. Gungor, "Performance of MAC protocols for wireless sensor networks in harsh smart grid environment," in Proc. 1st International Black Sea Conf. on Communications and Networking (BlackSeaCom), July 2013, pp. 50-53.

5. F. Aalamifar, L. Lampe, S. Bavarian, and E. Crozier, "WiMAX technology in smart distribution networks: Architecture, modeling, and applications," in Proc. IEEE PES Transmission and Distribution Conference and Exposition, 2014.

6. G. Iyer, P. Agrawal, E. Monnerie, and R. Cardozo, "Performance analysis of wireless mesh routing protocols for smart utility networks," in Proc. IEEE International Conference on Smart Grid Communications (SmartGridComm 2011), Oct 2011, pp. $114-119$.

7. D. Chen, J. Brown, and J. Y. Khan, "6LoWPAN based neighborhood area network for a smart grid communication infrastructure," in Proc. 5th International Conference on Ubiquitous and Future Networks (ICUFN 2013), 2013, pp. 576-581.

8. M. Islam, M. M. Uddin, M. A. A. Mamun, and M. A. Kader, "Performance analysis of AMI distributed area network using WiMAX technology," in Proc. 9th International Forum on Strategic Technology (IFOST 2014), 2014.

9. D. Bian, M. Kuzlu, M. Pipattanasomporn, and S. Rahman, "Analysis of communication schemes for advanced metering infrastructure (ami)," in Proc. 2014 IEEE PES General Meeting. IEEE, 2014, p. 5 pp.

10. A. Patel, J. Aparicio, N. Tas, M. Loiacono, and J. Rosca, "Assessing communications technology options for smart grid applications," in Proc. IEEE International Conf. on Smart Grid Communications (SmartGridComm). IEEE, 2011, pp. 126131.

11. S. Panchadcharam, G. A. Taylor, Q. Ni, I. Pisica, and S. Fateri, "Performance evaluation of smart metering infrastructure using simulation tool," in Proc. 47th International Universities Power Engineering Conference (UPEC 2012), 2012, pp. $1-6$.

12. G. Iyer, P. Agrawal, and R. S. Cardozo, "Performance comparison of routing protocols over smart utility networks: A simulation study," in Proc. 5th IEEE International Workshop on Management of Emerging Networks and Services (IEEE Globecom Workshops), December 2013, pp. 969-73.

13. B. Lichtensteiger, B. Bjelajac, C. Muller, and C. Wietfeld, "RF-Mesh systems for smart metering: system architecture and performance," in Proc. First IEEE International Conference on Smart Grid Communications (SmartGridComm 2010), 2010, pp. 379-384.

14. P. Kulkarni, S. Gormus, Z. Fan, and F. Ramos, "Ami mesh networks: A practical solution and its performance evaluation," Smart Grid, IEEE Transactions on, vol. 3, no. 3, pp. 1469-1481, 2012.

15. S. Elyengui, R. Bouhouchi, and T. Ezzedine, "LOADng routing protocol evaluation for bidirectional data flow in AMI mesh networks," International Journal of Emerging Technology and Advanced Engineering, vol. 5, June 2015.

16. Hydroquebec, 'Remote meter-reading project phase 1," online, "Regie de l'energie du Quebec", May 2012, http://publicsde.regie-energie.qc.ca/projets/34/ DocPrj/R-3770-2011-B-0157-TRAD-D0C-2012_05_23.pdf. 